\title{
Characterization of supercritical reactive flow for hypersonic real-time application
}

\author{
N Gascoin ${ }^{1}$ and P Gillard ${ }^{2}$ \\ PRISME, IUT Bourges, 63, avenue de Lattre de Tassigny - 18000 Bourges, France \\ M Bouchez ${ }^{3}$ \\ MBDA-France, 8 rue Le Brix - 18000 Bourges, France
}

\begin{abstract}
In the framework of the hypersonic propulsion, with cooled engine by endothermic hydrocarbon fuel, it is necessary to provide adapted measurement methods for the cooling regulation as for the control of the engine thrust. The sensors should be robust because of the extreme in-flight conditions, of vehicle acceleration and vibration, of the fluid temperature $(1500 \mathrm{~K})$ and pressure $(3.5 \mathrm{MPa})$ and of the multi-component supercritical mixture. Their response time should be lower than one second. Therefore, a large range of real time and on line measurement methods has been tested and some of these methods among the most promising are presented in this paper. The aim is not to necessarily develop a new technology but to evaluate the feasibility and the adaptability of existing ones for our purpose, even if those are not especially dedicated for it. Numerical evaluation is firstly conducted then some of the techniques are experimentally tested. Two of them, the sonic throat and the Fourier Transform Infra Red spectroscopy (FTIR), appear to be particularly promising and allow uncertainties of few percents. The first one allows evaluating the mass flow rate of a supercritical reactive mixture. The second one characterizes the chemical composition before injection in the combustion chamber, for mole fraction greater than $5 \%$.
\end{abstract}

\section{Introduction}

$\mathrm{H}$ ypersonic flight (over Mach 6) is expected to be achieved in the coming years by means of supersonic combustion ramjet (SCRamjet) [1]. For such high velocity, the total temperature of external air, considering the flow velocity, can reach $1650 \mathrm{~K}$ at Mach 6 and $4950 \mathrm{~K}$ at Mach 12. This produces a dramatic heating, which is added to the heat flux coming from the combustion heat release. Even composite materials could not withstand such large heat load. Furthermore, the time allocated for the combustion in SCRamjet mode is about $1 \mathrm{~ms}$. The thermal management of the overall vehicle and more specifically of the combustion chamber is thus an important focus for the aerospace research [2]. In this framework, the COMPARER program (COntrol and Measure of PArameters in a REacting stReam) has been settled by the MBDA-France company and by the Orléans University. It consists in studying the existing cooling strategy and in testing suitable measurement methods for the regulation. Indeed, an integrated solution using the fuel is considered. Hydrogen could be used as cooling fluid but due to safety and ease of storage reason, hydrocarbons are often preferred for flight Mach number under 8. The hydrocarbon fuel is injected in a channel equipped with pin fin. It surrounds the engine. In more efficient systems, the ceramic matrices composite are used [3]. A counter-flow heat exchanger is thus available towards the burned gases. When heated above $800 \mathrm{~K}$, the fuel is pyrolysed and thanks to its endothermic behaviour, it increases the active cooling of the hot walls. This pyrolysis produces lighter hydrocarbon species with shorter ignition delays than the initial fuel. This is particularly interesting for the combustion because of short residence time in the combustion chamber. It is important to note that the expected high pressure in the cooling loop ( $>3 \mathrm{MPa}$ ) causes the fluid to become supercritical in the channel. The internal wall of the cooling channel can be porous; creating a thin film cooling that contributes to protect it from the hot gases.

The complete system is a coupled process. The engine thrust depends on the chemical fuel composition injected in the combustion chamber but this composition is linked to thermal heat flux applied on the cooling channel

\footnotetext{
${ }^{1}$ Doctor, PRISME Institute, IUT Bourges, 63 avenue de Lattre de Tassigny, 18000 Bourges, France, AIAA Member

${ }^{2}$ Professor, PRISME Institute, IUT Bourges, 63 avenue de Lattre de Tassigny, 18000 Bourges, France.

${ }^{3}$ Research Engineer, MBDA-France, 8 rue Le Brix - 18000 Bourges, France, AIAA Member.
} 
depending on the air/decomposed hydrocarbon combustion. The latest is related to the combustion. Because of this interaction between combustion and pyrolysis, it is useful to have information during the flight about the fuel composition. Furthermore, to control the thrust and the cooling, the mass flow rate of the injected fuel is required. It is not equal to the one pumped out of the tank because of carbon deposit along the channel during the pyrolysis process and because of transpiration through the porous wall. Consequently, the COMPARER project aims at studying the hydrocarbon pyrolysis and a possible instrumentation thanks to a specific numerical modelling and to an experimental test bench.

This code, called RESPIRE (a French acronym for SCRamjet Cooling with Endothermic Fuel, Transient Reactor Programming), is a one dimensional program able to simulate the hypersonic vehicle cooling as well an academic chemical reactor heated by a furnace. It considers an existing detailed pyrolysis mechanism (1185 reactions, 153 species) [4] for the n-dodecane but it could treat other fluids with appropriate pyrolysis mechanism or with data tables giving the fluid properties [5]. The n-dodecane is used because of its purity and because it is representative of aeronautic kerosene. It is also used experimentally. The test bench is particularly suitable for hydrocarbon pyrolysis at supercritical state (1900 K, $8 \mathrm{MPa}$ ) under stationary conditions but it aims at studying transient test cases too [6]. Both of these tools have been used to identify some characteristic parameters of the pyrolysis phenomena [7], on which are based the measurement methods under study in this paper. Considering the mass flow rate, this parameter is the one to measure because its value is needed for the engine control. Relatively to the chemical characterization, a parameter has to be chosen : knowing the complete composition in real time is probably not mandatory for the control of an operational hydrocarbon-cooled engine. The estimated ignition delay is a good indicator of the fluid capacity to burn [7]. This delay can be related to the chemical composition and particularly to light species such as methane or ethylene [8]. Consequently, the measurement method should focus on the detection of some chemical species, which will then be related to ignition delay or to other combustion characteristic.

For the industrial use, the required accuracy is limited because the purpose is not to develop a specific technology allowing to know in details the process but rather to evaluate the ability of the existing ones and to use a commercial sensor. The originality of this study is to test on line methods in conditions, or for a purpose, for which they are not a priori dedicated. Rather than developing new technologies, it could be faster and cheaper to test some, for which the reliability is recognized. The present work uses an innovative approach because it involves in-depth numerical calculations to evaluate a wide range of methods before testing it on the experimental bench. As far as we know, there is no similar work in the literature.

We have shown in previous works that the chemical composition of pyrolysed mixture is complex; more than hundred of compounds are produced [7]. Furthermore, the fluid becomes supercritical because of the temperature and the pressure inside the cooling channel. This state of fluid is not yet well-known. Only few studies are available with measurements on it. These ones mainly consider $\mathrm{CO} 2$ or $\mathrm{H} 2 \mathrm{O}$ [9], which do not yield to difficulties such as carbon deposit or extreme operating conditions and these fluids are studied at temperature lower than $800 \mathrm{~K}$. That is the same for the study about some hydrocarbons [10], otherwise the measurement may not be conducted in real time, but a posteriori [11]. These studies do not aim at developing or testing measurement instruments but they only use them without modification.

Because of the extreme conditions of temperature ( $1500 \mathrm{~K}$ at least) and pressure (3.5 $\mathrm{MPa}$ at least), and because of the unknown chemical composition of the fluid, commercial mass flow meter are not able to operate, whatever their principle [12]. Due to the pyrolysis process inside the cooling channel, we have shown a probable high carbon deposit rate depending on the nature of the channel material [7]. This results in a jamming up which could disturb the instrument. Furthermore, a radial thermal gradient of about $40 \mathrm{~K}$ may appear inside the cooling channel [13]. This results in potential heterogeneity in the fluid flow, which makes the measure to be difficult. In addition, the instruments have to provide real-time on line measurements. They should be robust and compact enough in order to be used for hypersonic in-flight application, subjected to various vibrations of unknown frequency and strong acceleration, with limited mass and bulk requirements.

The aim of this paper is to present some of selected real-time measurement methods, which can be suitable for onboard applications under extreme conditions. The first category focuses on the mass flow rate whereas the second one is dedicated to the chemical characterization. The principles of these technologies will be presented such as their evaluation for stationary and transient solicitations, their accuracy and their suitability for an industrial use. The measurement of the fluid temperature in the cooling channel is possible thanks to an immersed thermocouple. 


\section{Mass flow rate measurement}

For standard applications, in the range of $100 \mathrm{~K}$ to $800 \mathrm{~K}$ and/or from $0.1 \mathrm{MPa}$ to $50 \mathrm{MPa}$, many mass flow sensors are commercially available. They are of great interest depending on the specificity of the application : the nature of the fluid and its phase, the possible multi-phases flow, the pressure and the temperature of the application, the environment conditions, the dynamics of the measure, the accuracy and the repeatability for example. Several mass flow meter technologies are adapted to liquid and gas phases, in particular the following ones : ultrasonic, Vortex, electromagnetic, Coriolis, thermal, volumetric or based on the pressure. However, these measurement instruments are not specifically designed for our application.

Several methods have been tested to evaluate their adaptability to the present purpose. For example, if the fluid temperature is known locally just before and just after a heating resistor, it should be possible to evaluate the mass flow rate with a calculation of thermal balance for local heat exchange. This requires measuring also the channel wall temperatures, which participates to local heat transfer. Nevertheless, this simple method is not suitable for our purpose because all the computations conducted on this point give a maximum error on the mass flow rate of a factor 2 [14]. This is absolutely disqualifying because the maximum uncertainties should be lower than around $10 \%$ of the initial mass flow rate. This percentage corresponds to the mass loss due to carbon deposit and filtration through the porous wall. The error related to this method is due to the complex heat transfers in geometry with pin fin, in which much more detailed temperature cartography should be available to accurately determine the local mass flow rate.

Other measurement methods have been numerically or experimentally tested at the laboratory. For example, a mass flow meter using the Venturi principle could be suitable for our purpose if some parameters are obtain thanks to data tables. The Venturi is mainly adapted to perfect fluids, weighing and uncompressible such as the non-viscous liquids. It allows linking the pressure loss to the flow speed thanks to the Bernoulli formula. This is a stationary method but due to its simplicity, it has been considered for this study. Nevertheless, the experimental tests have shown that a high accuracy, of few Pascal, on differential pressure is needed for the test configuration, with a mass flow rate of 0.1 g.s-1. The mass flow rate divided by the cross section area should not be higher on the actual scramjet. Thus, this would be the same problem for the industrial use. The required measure precision is estimated to be too high for our application even with differential pressure sensor. Other methods based on the pressure loss measurement (for example with unsteady measurement such as Vortex method) have also been tested but none of them seems very promising.

The following section provides a numerical and analytical study of the two most promising technologies considering all the limitation explained in the preceding part.

\section{A. The hot wire}

Considering the well-known principle of the hot wire [15] to determine the flow velocity, based on the Seebeck effect [16], it may be possible to obtain the mass flow rate. But first, it is necessary to know the density. For this purpose, the code developed in the framework of the COMPARER project has been used. Several various test cases have been considered with different specificities (Table 1) for an operating pressure of 3.5 MPa. The standard configuration corresponds to an academic chemical reactor inserted in a furnace but three calculations, with very high mass flow rate (over 60 g.s-1), are related to full calculations of the SCRamjet. The temperature profiles along the channel vary between the cases. The pyrolysis process is taken into account in all of these cases. So that, the chemical compositions of the fluid are different for all the computations because of the different length, mass flow rate and temperature profile.

Table 1. Test cases condition of reference numerical data.

\begin{tabular}{|c|c|c|c|c|c|}
\hline \multirow{2}{*}{$\begin{array}{l}\text { Test } \\
\text { case }\end{array}$} & \multicolumn{3}{|c|}{ Channel } & \multirow{2}{*}{$\begin{array}{l}\text { Maximum fluid temperature in the } \\
\text { reactor }(\mathrm{K})\end{array}$} & \multirow{2}{*}{$\begin{array}{l}\text { Mass flow } \\
\text { rate }\left(\mathrm{g} \cdot \mathrm{s}^{-1}\right)\end{array}$} \\
\hline & $\begin{array}{l}\text { Length } \\
\text { (m) }\end{array}$ & $\begin{array}{l}\text { Width } \\
(\mathrm{m})\end{array}$ & pin fins & & \\
\hline 1 & 2.2 & 0.212 & With & 1580 & 65 \\
\hline 2 & 2.2 & 0.212 & With & 1590 & 60 \\
\hline 3 & 0.6 & 0.008 & With & 1160 & 0.1 \\
\hline 4 & 1.2 & 0.008 & With & 1560 & 0.1 \\
\hline 5 & 0.9 & 0.04 & With & 1020 & 1 \\
\hline 6 & 1.1 & 0.212 & With & 1220 & 65 \\
\hline 7 & 1.2 & 0.002 & Without & 1520 & 0.2 \\
\hline 8 & 1.2 & 0.002 & Without & 995 & 0.05 \\
\hline
\end{tabular}


For these various different pyrolysis cases, the density is approximately a function of temperature, whatever the chemical composition (Figure 1a). The interesting point of this figure is to show the common behaviour of the tested configurations but not to study the discrepancies between them. Their similar results are particularly strong for high temperatures, over $1200 \mathrm{~K}$. Because this temperature will largely be exceeded near the cooling channel outlet in the SCRamjet, it can be concluded that giving the density in behalf of the temperature gives around $3 \%$ of uncertainty over $1200 \mathrm{~K}$. The mass flow rate is directly proportional to the density, so that there is the same uncertainty on the mass flow rate. This remains quite acceptable for the COMPARER project.
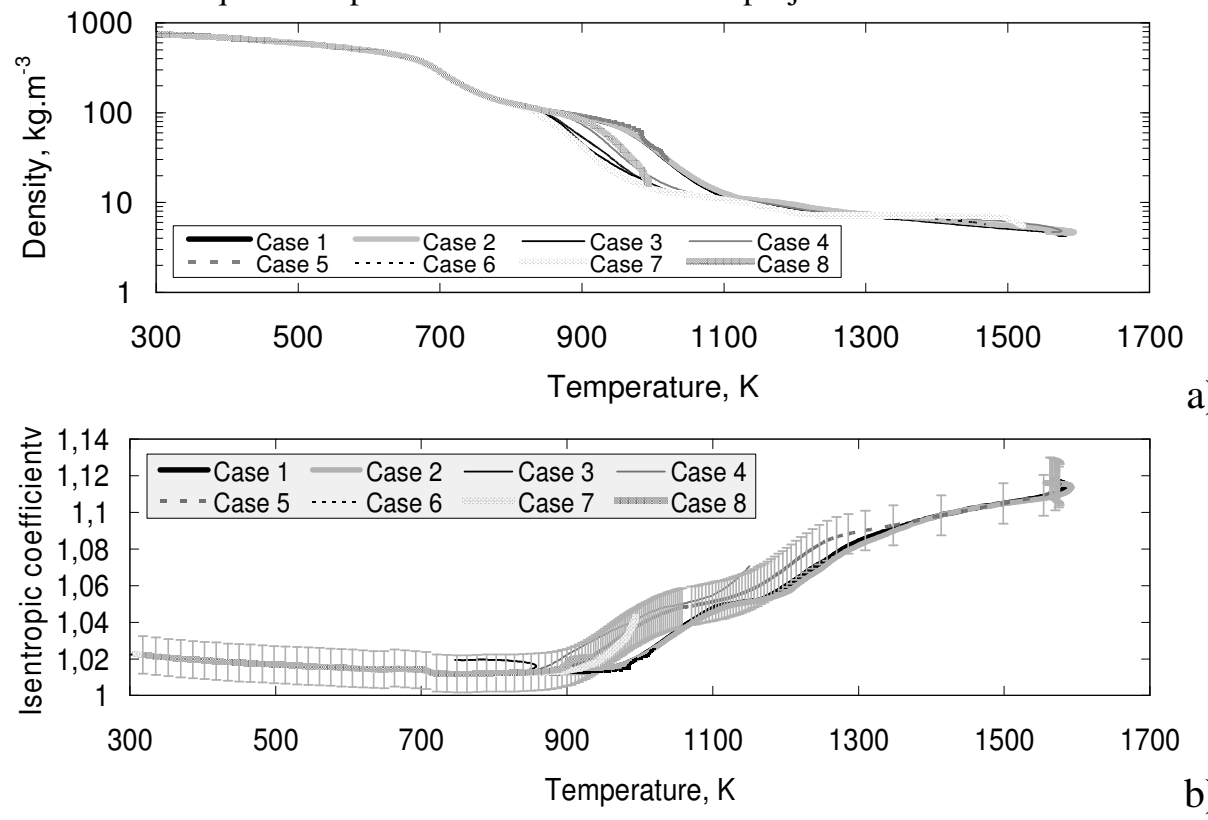

Figure 1. a) Density and b) Isentropic coefficient : both functions of temperature for all possible cases.

The frequencies of hot wire are well-known but it can also be computed. To obtain a sufficient accuracy, it is preferable to consider a hot wire at a much higher temperature than the fluid. If the wire, in tungsten, is heated electrically at a temperature around $2500 \mathrm{~K}$ for example, it is possible to compute its response time in the fluid flow depending on its diameter. The convective cooling of the wire governs the transient wire temperature (1) with the following constants $A=\frac{m_{\text {wire }} C p_{\text {wire }}}{h_{\text {wire }} S_{\text {wire }} T_{\text {fluid }}}$ and $B=\frac{1}{T_{\text {fluid }}}$ where $m_{\text {wire }}$ is the mass of the wire, $C p_{\text {wire }}$ its isobar heat capacity, $S_{\text {wire }}$ its convective exchange surface, $h_{\text {wire }}$ the convective exchange coefficient and $T_{\text {fluid }}$ the fluid temperature. Considering the variation of tungsten heat capacity with its temperature, thanks to the NIST database [17], three diameters are calculated for a given numerical flow case. For this test configuration, the channel wall temperatures are given, the fluid temperature is $1570 \mathrm{~K}$, the flow speed $27 \mathrm{~m} \cdot \mathrm{s}^{-1}$, the cross-section $5.3 .10^{-4} \mathrm{~m}^{2}$ and the convective coefficient $3800 \mathrm{~W} \cdot \mathrm{m}^{-2} \cdot \mathrm{K}^{-1}$ (Figure 2). A $10^{-5} \mathrm{~m}$ diameter seems to be sufficient for our study under transient use because its response time is much lower than one second. Its time constant is about $2.10^{-3} \mathrm{~s}$. This allows continuing the study of the hot wire on a more concrete aspect.

$$
T_{\text {wire }}(t)=\frac{1}{B}\left(1-\left(1-B \cdot T_{\text {wire,initial }}\right) e^{\left(-\frac{B . t}{A}\right)}\right)
$$

There are two possible ways to use a hot wire. In the first one with fixed intensity, the wire temperature is not constant. This modifies the electric resistance, which is measured. The second way is to fix the temperature thanks to a Wheastone bridge. The voltage is linked to the flow speed by the King's law [15]. A preliminary calibrating is required. Eq.(2) gives the wire electric resistance $R_{\text {wire }, T}$ at the temperature $T$ with $R_{\text {wire, } 300 \mathrm{~K}}$ the electric resistance at $300 \mathrm{~K}$ and $C_{T \text {, wire }}$ the temperature coefficient of the tungsten [18]. Its value corresponds to the absolute sensor accuracy and its derivative is the relative one. A wire diameter of $10^{-5} \mathrm{~m}$ presents a resistance comprised between $10 \Omega$ and $20 \Omega$, between $1570 \mathrm{~K}$ and $2500 \mathrm{~K}$. This is convenient for a sensor because there is no problem to measure it. 


$$
R_{\text {wire }, T}=R_{\text {wire } 300 \mathrm{~K}} \cdot\left(1+C_{T, \text { wire }} \cdot(T-300)\right)
$$

Considering a $10 \mathrm{~V}$ alimentation, an electric intensity of around $1 \mathrm{~A}$ should be applied to the wire. This value is necessary to heat the wire at a temperature near $2500 \mathrm{~K}$ and it is acceptable for the sensor. Furthermore, the intensity $I$ needed to compensate the wire cooling in the fluid flow is $0.15 \mathrm{~A}$ (3). The electric power $\mathrm{P}_{\text {elec }}$, resulting in thermal power through the Joule effect, is equal to the convective heat flux between the wire and the fluid. All these data are acceptable for in-flight applications.

$$
I^{2}=\frac{\mathrm{P}_{\text {elec }}}{R}=\frac{h_{\text {wire }} S_{\text {wire }}\left(T_{\text {fluid }}-T_{\text {wire }}\right)}{R}
$$

The sensor should also be interesting for transient measurement. Another numerical flow case, with the same channel wall temperatures, has been used with a slight different mass flow rate (almost $10 \%$ lower than the previous one). A mass flow rate gradient is thus considered. The outlet fluid temperature is assumed to decrease from $1570 \mathrm{~K}$ to $1556 \mathrm{~K}$. Thanks to previous equations, it is possible to determine that the hot wire needs $0.1 \mathrm{~s}$ to stabilize between these two temperatures. It corresponds to an intensity variation of $1 \%(0.15 \mathrm{~A})$. This is perfectly admissible for our purpose. Finally, it is previously required to calibrate the hot wire to measure the flow velocity. This calibration has to be done for various densities, whatever the chemical composition. Then, data tables should be used for appropriate density. As stated above, the latest is known during the process thanks to a function of the temperature. It may be also possible to measure it with ultrasonic methods if these are adapted to working and ambient conditions.

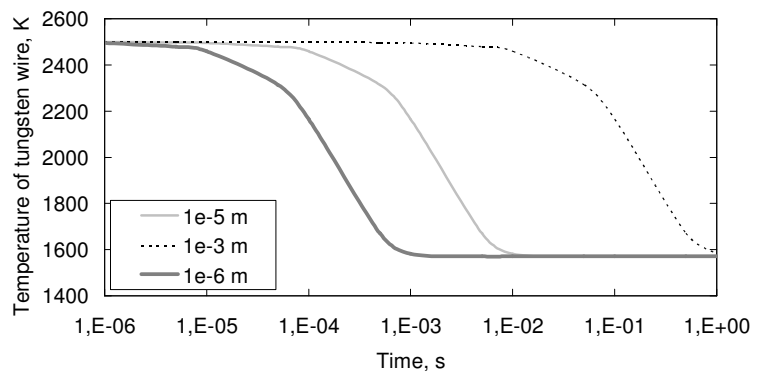

Figure 2. Transient temperature of tungsten wire immersed in a hot fluid in behalf of its diameter.

The technology of hot wire could be applicable to measure the mass flow rate by use of data tables giving the density as a function of the fluid temperature. The wire has the advantage to be compact and probably robust enough. A technical solution has even been thought to directly integrate such a sensor in the conception of the cooling channel with thermal and electric insulation towards this one. Nevertheless, the hot wire has some drawbacks that have to be taken into account. It is not suitable for biphasic flow, which in fact is not the case in our supercritical conditions. This intrusive technology may locally increase the pyrolysis process in the surrounding boundary layer because its temperature is over the one of the fluid. Furthermore, if carbon deposit appears on the hot wire, due to coking activity during the pyrolysis, it could disturb the heat transfer between the fluid and the wire. This would results in an approximate measure, which is now difficult to anticipate by numerical calculations. It has to be noticed that the principle of the hot wire could also be used with a cooled wire, which gives the same advantage but without the local pyrolysis issue. The cooling of the wire could be obtained thanks to the Peltier effect [16]. For a diameter of $10^{-5} \mathrm{~m}$, the response time is evaluated to be $10^{-2} \mathrm{~s}$ in order to have a stabilization of the wire from $1000 \mathrm{~K}$ to $1570 \mathrm{~K}$.

\section{B. The sonic throat}

In the minimal section of the cooling channel, the flow velocity is exactly sonic if the downstream pressure is at least 0.528 lower than the upstream one (for an isentropic coefficient of 1.4). This is notably the case in the cooling channel of the scramjet because the operating pressure is higher than $3.5 \mathrm{MPa}$ and because the fluid is injected in the combustion chamber at a pressure around 0.2 MPa. Consequently, it may be possible to obtain the mass flow rate $\dot{m}$ in this minimal section $S_{\text {throat }}$ thanks to $P_{\text {tot }}$, the total pressure (4) [19], where $c^{*}$ is the characteristic velocity. Because the static pressure $P_{\mathrm{s}}$ is easier to measure, (5) is used with a Mach number $\mathrm{M}$ of 1 in the throat, where $\gamma=\frac{C_{\mathrm{p}}}{C_{\mathrm{v}}}$ is the isentropic coefficient with $C_{\mathrm{v}}$ the isochoric heat capacity. It is thus required to compute the 
characteristic speed (6), which is the inverse of the flow rate coefficient $C_{\mathrm{D}}$, with $r=\frac{\mathrm{R}}{M}$ the fluid constant, $\mathrm{R}$ the perfect gases constant and $M$ the molar mass. In theory, the sonic throat is adapted to perfect gases flow with constant isobar heat capacity. It is thus required to test it in the specific conditions of our application.

$$
\begin{gathered}
\dot{m}=P_{\text {tot }} \cdot S_{\text {throat }} \cdot \frac{1}{c^{*}} \\
\frac{P_{\mathrm{s}}}{P_{\text {tot }}}=\left(1+\frac{\gamma-1}{2} \cdot \mathrm{M}^{2}\right)^{\frac{-\gamma}{\gamma-1}} \\
C_{\mathrm{D}}=\frac{1}{c^{*}}=\left(\frac{2}{\gamma+1}\right)^{\frac{\gamma+1}{2(\gamma-1)}} \times\left(\frac{\gamma}{r . T}\right)^{0.5}
\end{gathered}
$$

The static fluid temperature is required to determine the characteristic velocity. It can easily be measured at the minimal section with a thermocouple. Thus, it is only needed to determine the isentropic coefficient $\gamma$ and the fluid constant $r$. These two parameters have been linked to the fluid temperature in order to build data tables. They have been calculated for several numerical cases, which correspond to the conditions given by the table 1 . The isentropic coefficient (Figure 1b) appears to be mainly a function of the temperature, whatever the chemical composition, the residence time inside the cooling channel or the applied heat fluxes. The error bars correspond to $\pm 1 \%$. This allows considering the isentropic coefficient as a function of temperature over a wide range, almost from $300 \mathrm{~K}$ to $1500 \mathrm{~K}$. Consequently, it is possible to use data tables based on measured static temperature. The same is observed for the fluid constant $r$.

Thus, the mass flow rate is known, thanks to the above equations, as a function of temperature for all the test cases. As a result, a sonic throat is now numerically simulated. The mass flow rate obtained for the eight cases have been divided by the cross section area of the throat. They are plotted for a wide temperature range from $300 \mathrm{~K}$ to $1600 \mathrm{~K}$ (Figure 3). The ratio between mass flow rate and cross section is directly linked to the temperature for all the calculations, despite the fact that the test cases are of various nature : length and width of the channel, with or without pin fins geometry, boundary temperature profile, mass flow rate. This is particularly promising for our measurement method but it was based only on computations. Furthermore, the results of Fan et al. [20] have afterwards been added on the figure to compare our numerical results about pyrolysed mixture based on n-dodecane to their experimental measures on Chinese kerosene. Due to the chemical discrepancies between the fluids, the trends are comparable but not from a quantitative point of view. Nevertheless, this is very encouraging. The results are good enough to be confident for fluid temperature higher than $700 \mathrm{~K}$ and for pressure over 3.5 MPa.

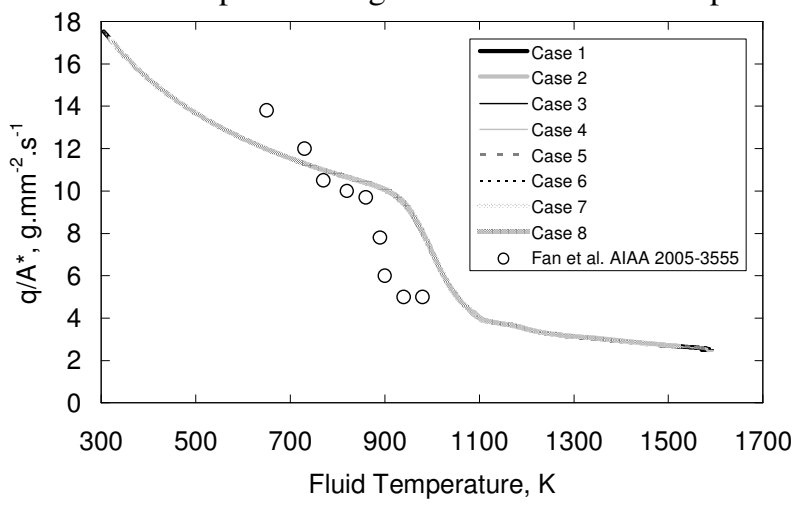

Figure 3. Numerical mass flow rate computation for different test cases (3.5 MPa).

The advantage of this non intrusive measurement method is its simplicity because it only needs to have a controlled minimal section and a measure of the static pressure and temperature at the throat. There is no chemical perturbation and the accuracy is about $1 \%$, which is better than the hot wire. The crippling due to carbon deposit could be a problem if it disturbs the temperature and pressure measurements or if it reduces the section in an important manner. Nevertheless, this method is estimated to be very promising.

It is planned to test it on the COMPARER test bench, in the middle of 2008, at pressure and temperature as high as $6 \mathrm{MPa}$ and $1500 \mathrm{~K}$. For the industrial use, such a sensor is compact and robust; its response time is roughly the one of temperature and pressure measurements. It could be preferably implemented near the injection hole, between the cooling channel and the combustion chamber. These holes present the minimal section of the cooling channel. Adding another throat upstream in the channel could lead to flow control problem (transient behavior of two ducts). 


\section{Characterising measure on the chemical composition}

Depending on the application, several analysis instruments are commercially available for very accurate measure on chemical compositions. For example, the mass spectrometry (MS) [21] allows identifying and quantifying all the components in a mixture, even some part per billion ( $\mathrm{ppb}$ ). But it is better if coupled to a compound's separation system such as a Gas Chromatograph. Unfortunately, this is rather a laboratory apparatus because the time required to analyze a sample containing heavy hydrocarbons is at several minutes. Vacuum pumps are also needed and this does not correspond to an onboard application. For in-flight application, this is not acceptable because a typical flight time is about twenty minutes. This apparatus is not dedicated for transient measurement and for control purpose. Analysis methods, such as Gas Chromatograph [22], are also not suitable for the same reason as the Mass Spectrometry and because of high retention time in the separation columns. Consequently, another kind of technology has to be used in order to characterize the chemical composition on a fuel-cooled system but with a probable lower accuracy.

Because the measurement method should be suitable for transient use, optical methods have been evaluated. These can be passive and non-intrusive, they have a good response time (about one second) and they do not disturb the fluid flow. The only drawback could be the crippling of optical windows with the carbon deposit or with other heavy chemical species. A solution could be to protect these windows with a very thin and controlled nitrogen flow between the pyrolysed fuel and the window. Such technique is used for particle size analyzers measurement for example [23] and has been tested in SCRamjet engine with limited air or nitrogen film injection [24].

In the optical technologies, the spectroscopic ones are the most interesting because they are common, well known and suitable for transient measurement [25]. Even if their size are still quite important, some new compact apparatus are now even available with integrated optical lens, which is interesting for in-flight applications. The active methods, which stimulate the species, are in particular useful for low temperature flows with low signal or for very diluted mixture [26]. For example, the CARS (Coherent Anti-Raman Stokes) technology is used to follow traces in combustion. However, for measurement in pyrolysed mixture at temperature up to $1500 \mathrm{~K}$, and for pressure higher than 3.5 MPa, the passive methods have proved to be sufficient [10]. The Infrared spectroscopy has also been used by MBDA-France and its partners in the RAPIERE project to evaluate the combustion efficiency in the combustion chamber [24]. Thus, it is suitable for online onboard real-time measurement and in an industrial test bench. Furthermore, the wavelength range to study has to be chosen. It is well known that hydrocarbons are visible in Infrared range but not in Ultra-Violet because they are fluorescent in this range [27]. This is the contrary for hydrogen. The hydrogen is not studied in this paper because it does not represent a great interest, as its production is limited to few percent in mass. This represents molar fractions in the pyrolysed mixture lower than $10 \%$. It has to be noticed that the hydrogen quantification has been treated in previous paper [28]. It is possible by means of thermal conductivity notably to detect and to quantify it [7].

Infrared spectroscopy (FTIR) are used since a tenth of year as control tool in industrial plants in particular. FTIR is often linked to thermogravimetric analysis (TGA) to study decomposition phenomena, such as pyrolysis process [29]. It is even used as a quantitative instrument [30]. The spectroscopic quantification methods are often based on the Beer-Lambert law and they are commercially available with instruments but this requires a preliminary calibrating under the condition of study. Thus, this is the main limitation of present techniques because it needs a long and complex calibrating. For our application, it could certainly be difficult to realize a calibrating on more than one hundred of species for conditions from $2 \mathrm{MPa}$ to $6 \mathrm{MPa}$ and from $800 \mathrm{~K}$ to $1500 \mathrm{~K}$ for example. The time required to obtain an infrared spectrum of a mixture is estimated to be around one minute with DTGS (Deuterated Triglycine Sulfate) detector and about one second with a MCT (Mercury Cadmium Telluride) one. Furthermore, it is difficult to quantify more than a tenth of species because it requires to select a spectral zone for each chemical compounds, preferably without interactions with others. Nevertheless, FTIR could be used to only quantify the main species, all the others could be grouped in families such as: alkenes, alkanes, cyclic, aromatic.

We present, in this section, the numerical exploitation that has been performed with Infrared spectra. The calculations have been conducted with the HITRAN software [31]. It is based on experimental database on molecular emission and it considers about twenty species over a wide range of temperature and pressure. The hydrocarbon ones are $\mathrm{CH}_{4}, \mathrm{C}_{2} \mathrm{H}_{2}, \mathrm{C}_{2} \mathrm{H}_{6}$. No interpolation is provided between the data points. HITRAN is dedicated to atmospheric studies even if an extrapolation is provided at higher conditions. The high temperature version of HITRAN, over $1000 \mathrm{~K}$, is called HITEMP. More information about calculation methods and about uncertainties is given in Ref. [32]. Extrapolation from ambient pressure and low temperature of the existing database is questionable in our supercritical high conditions but this does not change the qualitative interest of the following study.

It is expected to link the Infrared spectra to the combustion process. This has been previously published [8] and it has been shown that to ensure a correct mixture combustion, a minimum concentration of some species is required. 
It could thus be needed to evaluate the quantity of these species in the mixture. Some authors have used spectroscopic approach to determine a fluid composition but on samples with limited number of components [3335]. It uses the fact that each chemical chain, and even each chemical species, can be identified by the wavelength corresponding to various excitation modes. For example [34], the ethane presents a vibration mode at $930 \mathrm{~cm}^{-1}$ whereas the acetylene has a one near $850 \mathrm{~cm}^{-1}$ and the methane at $4170 \mathrm{~cm}^{-1}$. The methane has a maximum signal at $2927 \mathrm{~cm}^{-1}$ approximately, whereas the maximum for acetylene is $3340 \mathrm{~cm}^{-1}$.Various similar wavelengths exist for the same vibration mode of different compounds.

A parametric study has been conducted, in particular, to verify the use of the software. This point is detailed because it is necessary to identify the spectral signal of the fluid to propose a measurement method based on this point. The computation conditions are various enough to ensure a good diversity of study (Table 2).

Table 2. Parameters of spectra computations with the HITRAN software.

\begin{tabular}{ccccc}
\hline \hline $\begin{array}{c}\text { Species under } \\
\text { study }\end{array}$ & $\begin{array}{c}\text { Concentration in binary and } \\
\text { ternary mixtures }\end{array}$ & Sample thickness (m) & $\begin{array}{c}\text { Temperature } \\
(\mathrm{K})\end{array}$ & $\begin{array}{c}\text { Pressure } \\
(\mathrm{MPa})\end{array}$ \\
\hline $\mathrm{CH}_{4}$ & $0 \%-33 \%-50 \%$ & 0.01 & 900 & 3.5 \\
$\mathrm{C}_{2} \mathrm{H}_{2}$ & $66 \%-100 \%$ & 0.05 & 1200 & 7.0 \\
$\mathrm{C}_{2} \mathrm{H}_{6}$ & & 1 & 1500 & \\
\hline \hline
\end{tabular}

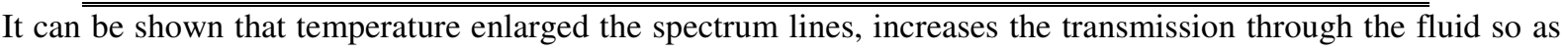
the emission of the fluid. This is due to the thermal excitation of molecules, which vibration modes expand on wider spectral lines. The pressure has the same effect for the spectrum width but it is slightly weaker. It is not for the same reason. Indeed it is attributed to the density increase. This is confirmed by the study of sample thickness and by the sample dilution effects. The signal of diluted sample can also be influenced by the other added species. The spectrum of a multi-compounds mixture shows a spectrum composed of the one of all the various species. It is still required to evaluate in which ways the mixture spectral signal is the "sum" of the pure component signals. It has to be noticed that the spectrum resolution is also an important point because a very fine analysis of some specific lines needs a high resolution (lower than $4 \mathrm{~cm}^{-1}$ ).

Thanks to the above considerations, it has been found with HITRAN that a wavelength of a specific spectral line can be followed to evaluate a species concentration even if other species are present in the mixture. An example is given in emission for the ethane at two temperatures over a wide range of molar fraction (Figure 4). This shows, for $1200 \mathrm{~K}$ and $1500 \mathrm{~K}$, a slight displacement of the wavelength depending on the quantity of ethane. This could be an interesting parameter to measure in order to evaluate the quantity of ethane. The accuracy is better for small amounts, under $50 \%$. The signal is relatively insensible when the concentration is below $20 \%$. For a molar fraction approaching $0 \%$, the spectral line does not become zero because it is obvious that even for a single particle, the vibration mode has a given wavelength. This result is shown for dilution in other hydrocarbon species but it is difficult to numerically confirm it because HITRAN does not consider other compounds. Two fitting equations are proposed for the two temperatures but it would be better to have only one, which considers the temperature as a parameter. Nevertheless, this method requires a high spectral resolution for high molar fraction, which is a bit penalizing. Furthermore, it appears to be difficult to quantify the ethane for molar fraction lower than $5 \%$ to $10 \%$. This is the limitation of the method but it is acceptable for the COMPARER project because the aim is to analyze the major species. The accuracy is estimated to be of few percent.

A similar approach has been undergone around $3140 \mathrm{~cm}^{-1}$. Instead of studying the wavelength of the vibration mode, the luminance signal is integrated over the wavelength range (Figure 5). The spectral peak width is used. A higher temperature, $1500 \mathrm{~K}$ (on the right scale) instead of $1200 \mathrm{~K}$, gives a better sensibility. The results are interesting, in particular for high concentration of ethane over $50 \%$, because of the sufficient sensibility of the method. It could be possible to combine a method based on the spectral line position, around $910 \mathrm{~cm}^{-1}$, and another one based on luminance integration around $3140 \mathrm{~cm}^{-1}$. This would allow evaluating the ethane quantity in various pyrolysed mixture. The methane and the acetylene have also been studied and such conclusions have been shown. This method can be extended to multi-species mixture only if the other components do not have exactly the same infrared spectrum. This is the case, even for similar hydrocarbons, because the vibration mode of a given chemical chain has a wavelength, which is function of the compound molar mass. Finally, the infrared spectroscopy is able to furnish information about chemical concentration for some major species but not for chemical traces, except in transmission with long optical length. The n-dodecane pyrolysis at high temperature produces mainly methane and ethylene. Information about these components could be sufficient for the chemical characterization, so that for the engine control. This technology is suitable for onboard, inline real-time measurement because considering only few species decreases highly the post-treatment time. Furthermore, this method does not need a calibration under the conditions of use because of the limited accuracy, which is wanted. 
The work is going on with experiments on IR spectroscopy. The real-time quantification of gaseous pyrolysis products is now validated, since the beginning of 2008, by comparison with GC/MS data.

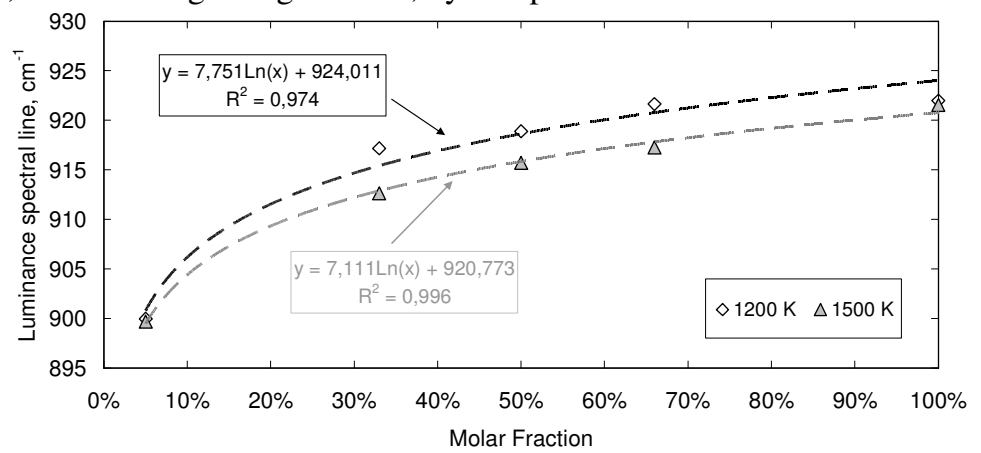

Figure 4. Relationship between the ethane concentration (in various mixtures with methane and acetylene) and the exact wavelength position of the first main vibration mode.

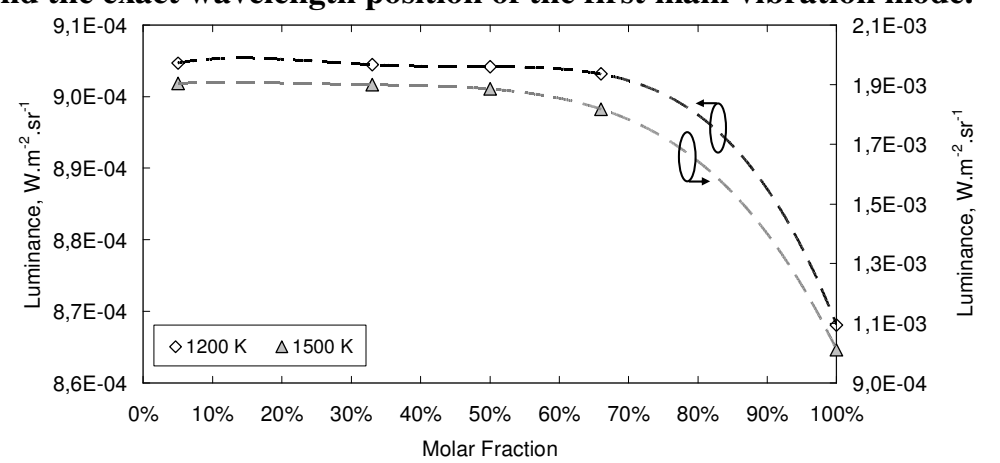

Figure 5. Relationship between the ethane concentration and the luminance of a vibration mode around $3140 \mathrm{~cm}^{-1}$.

\section{Conclusion}

The COMPARER project has been settled in the framework of the cooling strategy, adopted for the thermal management of the scramjet. It aims at studying the phenomena and their interactions related to the fuel pyrolysis before identifying some characteristic parameters. The final purpose is to propose adapted real-time and on line measurement methods for in-flight application. These methods should focus on the mass flow rate measurement and on the chemical characterization of the pyrolysed fluid. Due to the extreme difficulties encountered in the fluid flow (high temperature, supercritical state, multi-species, coke formation) and in onboard conditions (vibrations and acceleration of the vehicle), it has been chosen to evaluate the appropriateness of existing measurement methods. As far as we know, there are no measure instruments directly suitable for our purpose. A systematic approach has been used to test the suitability of various existing technologies. It has been verified if these ones are adequate to in-flight transient use. Two promising solutions have been kept. The first one, the sonic throat, enables measuring the mass flow rate and it has an uncertainty of about $1 \%$. The second one, the Infrared spectroscopy, chemically characterizes the pyrolysed mixture.

The numerical study has demonstrated that these technologies are interesting ways. Even if the FTIR spectroscopy could not be used to determine the exact composition of the fluid, such as a Gas Chromatograph for example, it could give the proportion of some major light species in the mixture. The minimum quantification is about $5 \%$ to $10 \%$ in mole. The post-treatment is limited so that a quantitative measurement could be furnished in about one second. This would furnish information about the mixture combustion ; notably by relating the measures to ignition delay.

The 4 years study presented in this paper has allowed to identify the promising measurement methods, which will be developed in the second phase of the COMPARER program, until end of 2009. A specific test cell has been designed and is used to evaluate experimentally the sonic throat and the IR spectroscopy. This one has already been tested and no leaks are noticed even at $8 \mathrm{MPa}$ and more than $900 \mathrm{~K}$. The experiments are expected to be done in middle 2008. Some IR experiments have already been conducted and these have been of great interest for the comprehension and the test of feasibility. The information obtained with this technology should be sufficient to chemically evaluate the fluid flow. 


\section{Acknowledgments}

The present work was realised with the contribution of the "Conseil Général du Cher", of the "Conseil Régional du Centre", of the FRED, of the FEDER, of the FSE, of MBDA-France and of Y. Parmantier.

\section{References}

[1] Fry R S, 2004, A century of ramjet propulsion technology evolution, J. of Prop. and Power, Vol. 20 No. 1 Jan.-Feb. 27-58

[2] Falempin F and Serre L, 2004, LEA flight test program - status in 2004, 40th AIAA Joint Propulsion Conference And Exhibit, AIAA-2004-3344, Fort Lauderdale, Florida, 11 - 14 July 2004

[3] Bouchez M and Beyer S, 2005, Ptah-socar fuel-cooled composite materials structure for dual-mode ramjet and liquid rocket engines - 2005 status, 13th International Space Planes and Hypersonics Systems and Technologies, AIAA 2005-3434

[4] Dahm K D et al., 2004, Experimental and Modelling Investigation of the Thermal Decomposition of n-Dodecane, J. Anal. Appl. Pyrol. 71 865-81.

[5] Gascoin N, Gillard P, Dufour E and Touré Y 2007 Validation of transient cooling modeling for hypersonic application, Journal Of Thermophysics And Heat Transfer Vol. 21 N 1 Jan-Mar 2007 86-94

[6] Gascoin $\mathrm{N}$ et al., 2006, Measurements for fuel reforming for scramjet thermal management and combustion optimization : status of the COMPARER project, 14th Hypersonic Systems and Technologies Conference, Canberra, Australia, 6-9 Nov.

[7] Gascoin N 2006 Etude et mesure de paramètres pertinents dans un écoulement réactif Ph.D. Thesis available at http://tel.archives-ouvertes.fr/tel-00128418 Orleans University 30/11/06 Bourges

[8] Daniau E, Bouchez M, Herbinet O, Marquaire P M, Gascoin N and Gillard P 2005 Fuel reforming for scramjet thermal management and combustion optimization 13th AIAA/CIRA Hypersonic Conference Capua (Italy) 16-20 May AIAA 2005-3403

[9] Bazaev A R et al., 2003, PVTx measurements for H2O+D2O mixtures in the nearcritical and supercritical regions J. of Supercritical Fluids 26 115-28

[10] Chen X, Yasuda K, Sato Y, Takishima S and Masuoka H 2004 Measurement and correlation of phase equilibria of ethylene+n-hexane+metallocene polyethylene at temperatures between 373 and 473K and at pressures up to 20MPa Fluid Phase Equilibria 215 105-15

[11] Zhao S, Xu Z, Xu C, Chung K H and Wang R 2005 Systematic characterization of petroleum residua based on SFEF Fuel $84635-45$

[12] Gailledreau C Choix d'un débitmètre Techniques de l'Ingénieur R2200

[13] Gascoin $\mathrm{N}$ et al., 2006, Numerical and experimental validation of transient modeling for scramjet active cooling with supercritical endothermic fuel, 4th IECEC Congress, San Diego 26-29 june 2006 AIAA 2006-4028

[14] Gascoin N, Gillard P, Bernard S, Touré Y, Daniau E, Bouchez M Les méthodes de mesure COMPARER Internal technical document COMPARER Project September 200641 pages

[15] Schon J P, Comte-Bellot $\mathrm{G}$ and Charnay G Anémomètres à fil ou à film chaud Techniques de l'Ingénieur R2272

[16] Bonnier G, Devin E, Couples thermoélectriques: Caractéristiques et mesure de température Tech.Ing. R2590

[17] NIST Chemistry Webbook http://webbook.nist.gov/chemistry/ acceded in March, the third 2007

[18] Jones 2000 Langmuir Bulletin de l'Union des Physiciens 11/2000

[19] Bouchez M Propulsion par statoréacteur Third Year ESEM courses Energetic and Thermal Science speciality Orléans

[20] Fan X, Yu G and Li J 2005 Flow rate and calibration of kerosene cracking for supersonic combustion AIAA 2005-3555

[21] Chapman J R 1993 Practical organic mass spectrometry 2nd Edition Wiley Interscience John Wiley \& Sons New York

[22] Grob R L 1995 Modern practice of gas chromatography Editions Wiley Interscience Third Edition

[23] Malvern Instruments 2007 Instruments for material characterisation http://www.malvern.co.uk acceded the 08/04/2008

[24] Minard J P et al., 2002, RAPIERE: An innovative industrial optical measurement system for scramjet flows (first phase project final status in 2002), 11th International Conference Space Planes and Hypersonic Systems and Technologies, 29/09 04/10 2002 Orléans FRANCE AIAA-2002-5156

[25] Rouessac A 1992 Analyse chimique : méthodes et techniques instrumentales modernes Masson

[26] Hollas J M 1987 Modern spectroscopy J. Wiley \& Sons

[27] Colthup P B, Daly L H and Wiberly S E 1990 Introduction to infrared and Raman spectroscopy Academic Press

[28] Gascoin $\mathrm{N}$ et al. 21/10/2004 Mesures et contrôle de paramètres pertinents pour la régulation d'un statoréacteur mixte refroidi Troisième Rencontre Capteurs Bourges

[29] Bassilakis R, Carangelo R M and Wojtowicz M A, 2001, TG-FTIR analysis of biomass pyrolysis, Fuel 80 1765-86

[30] van de Voort F R, Sedman J, Cocciardi R and Juneau S An automated FTIR method for the routine quantitative determination of moisture in lubricants: An alternative to Karl Fischer titration Talanta doi:10.1016/j.talanta.2006.10.042

[31] Rothman L S et al. 1998 The hitran molecular spectroscopic database and hawks (hitran atmospheric workstation): 1996 Edition J. Quant. Spectrosc. Radiat. Transfer Vol. 60 No. 5 665-710

[32] Rothman L S The HITRAN database Harvard-Smithsonian Center for Astrophysics http://cfawww.harvard.edu/HITRAN/ accessed the 08/04/2008.

[33] Umber, J Spectroscopie infra rouge, Organic mineral and structural chemistry courses, Académie de Nancy-Metz http://www.ac-nancy-metz.fr/enseign/physique/chim/Jumber/Default.htm accessed the 08/04/2008

[34] Dalibart M, Servant L. Spectroscopie dans l'infra rouge Techniques de l'Ingénieur P2845

[35] Malow M, Schulze A and Krause U, 2006, The main gaseous products and the mass loss during the self-ignition of combustible materials, Proceedings of Sixth ISHPMIE Vol. III Dalhousie University Halifax NS Canada Aug 27-Sept.1 825-36

10

American Institute of Aeronautics and Astronautics 